\title{
Parametric model updating with frequency and MAC combined objective function of port crane structure based on operational modal analysis
}

https://doi.org/10.1515/phys-2019-0008

Received October 29, 2018; accepted January 28, 2019

\begin{abstract}
In FE modeling of port crane structures, errors may be caused due to the simplification of complicated components, unclear physical properties, local approximation and inaccurate boundary conditions. So it is very important to update the FE model accuracy according to the experiment model and results. However there are two major problems when solving the model updating of port crane structure, the one is the difficulty of collecting the structure modal parameters with traditional modal experiment method because of the large scale of port crane structure, the other is mismatching problem while updating processing due to most of the model updating method currently constructing the objective function only by frequency. In this paper, an operational modal analysis is adopted to estimate the modal parameters of port crane structure based on the operational condition. A parametric model updating methodology of finite element (FE) model is proposed to solve port crane structure model updating: a series of design parameters of the FE model of port crane structure are selected as the main factors based on the sensitivity analysis, the objective function is built by considering the frequency correlation and the modal assurance criterion (MAC) together, and finally a zero-order/first-order combination algorithm is utilized to get the final accurate FE model of port crane structure. Research results showed that methodology proposed in this paper has satisfied updating accuracy for port crane structure, which verifies that the proposed method is suitable for model updating problem of this kind of structure.
\end{abstract}

Keywords: Parametric model updating, sensitivity analysis, port crane structure, operational modal analysis.

PACS: 02.10.-v; 06.20.fb; 06.90.+v

\section{Introduction}

In recent years, the automated container terminal (ACT) has had rapid development, and also a lot of research work for example intelligent decision and machine vision, have been carried out to further help this development [1-5]. Port cranes are the main equipments at ACT for loading and unloading, and their dynamic properties will have direct influences on efficiency and safety of operation. With the rapid development of ACT, the port cranes are becoming large-scale, high speed, heavy loaded and automatic, which poses higher requirements for port crane structures. Further researches in the model updating problems of port crane structures based on the operational modal analysis make it possible to establish accurate dynamic models with finite element method of the port crane structures under all kinds of working conditions, which can reflect the structure dynamic properties accurately and offer the orientation of structure optimization and reference for positive and negative control of port crane structures.

An accurate dynamic model of large port crane structure can accurately reflect the dynamic characteristics of structure, help the structure design for disaster prevention and reduction, indicate the direction for structure optimization and provide the basic information for online structure monitoring and damage identification. Traditionally, the dynamic model of structure is obtained by an experimental modeling method or theoretical modeling methods (mainly refers to the FEM), in practice, updated structural dynamic model is usually obtained by the FE model updating according to the experiment model.

Structural dynamic analysis of the experimental model is often referred to get structure modal parame-

\footnotetext{
*Corresponding Author: Weiguo Zhang: Shanghai Offshore Engineering Research Institute, Shanghai Maritime University, Shanghai, 201306, China, E-mail: wgzhang@shmtu.edu.cn

Octavian Postolache: Inst Telecomunicacoes, Lisbon, Portugal and Inst Univ Lisboa ISCTE IUL, Lisbon, Portugal,

E-mail: octavian.postolache@gmail.com
} 
ters by the modal analysis method. This modal analysis method is based on experiment and can be divided into traditional experimental modal analysis method and operational modal analysis method. For large engineering structure such as a port crane, it is really difficult to excite the structure effectively. So usually it can't getthe frequency response functions (FRFs) of the structure, which means it is impossible to estimate the system modal parameters by the traditional experimental modal analysis. As a matter of fact for port crane structure, it's normally easier to get the structure outputs rather than the inputs, in that case, the operational modal analysis is a valid and necessary method to estimate the structure modal parameters by the output data only. Compared with the traditional experimental modal analysis method, the operational modal analysis has obvious advantages: the structure is excited only by the ambient excitation or the machine itself, the test cost is low; Since no artificial equipment is used for the excitation, there will be no local damage to the structure and the structure is safe; As the output of system response is closer to the vibration response when the structure is operating, therefore, the identified modal parameters are more in line with the reality and boundary conditions.

Model updating has been widely used in aerospace, civil engineering, mechanical engineering and other fields [6-11]. Distinguishing from the updating object, the FE model updating methods can be divided into matrix model updating method [12,13] and parametric model updating method [14]. Because of matrix model updating method often leads to the mass matrix and stiffness matrix of the structure losing physical sense, it has been gradually replaced by the parametric model updating method. The basic idea of parametric model updating method is combined with optimization problems. By constructing the error (objective function) of the modal parameters between theoretical model and experiment model under the same conditions, specific updated parameters are then selected to update and minimize errors to achieve the purpose of correction. The objects of the parametric model updating method are the design parameters of the FE model directly which makes the adjusted results have definite physical meaning, and meanwhile it can be used to locate the model error, so this method is the mainstream of today's FE model updating method $[15,16]$. but there are still some problems that will be encountered when adopting the parametric model updating method into the engineering applications, the major one is mode shape may be miss-paired during updating processing, because the objective function is usually constructed only by the fre- quency instead of considering frequency and mode shape together.

In this paper, the modal parameters of port cranes are estimated by operational modal analysis, a multi-objective optimization model is constructed considered with frequency and MAC values together, the main factors of the physical parameters are determined by the sensitivity analysis, and the optimization model is minimized by the zero-order/first-order combination algorithm.

\section{Operational modal analysis of port crane structure}

Operational modal analysis assumes that the input signals are white noise sequences, on this assumption, the system is an input-output one, which means the inputs are white noise sequences, and the outputs are the system responses. In fact, the natural excitation is always color noise, but we can still have the satisfied identification results as long as the inputs are stationary stochastic processes, and which frequency bands can cover the structure's bands which we interested.

By now, there are several famous methods for identifying the operational modal parameters of the large scale structure based on the natural excitation (e. g. natural excitation technique (NEXT), stochastic subspace identification (SSI), PolyMax etc.) [17, 18]. Because the advantages of PolyMax are that: it is poly-reference, fast, numerically stable for large-bandwidth with high-mode order analysis, and yields very clear stabilization diagrams even with highly noisy FRFs measurements [19], it is widely adopted to identify the modal data with the operational conditions.

PolyMax is a dedicated frequency-domain algorithm for dealing the huge amount of output-only data. When system input is the white noise, port crane structural responses spectral function has the similar expression as the frequency response function, and then the structure modal parameters can be identified by estimating the responses spectral model.

During port crane structure operational modal analysis, the power spectra of the outputs can be expressed as

$$
\left[S_{X X}(\varpi)\right]=[H(\omega)]\left[S_{F F}(\varpi)\right][H(\omega)]^{H}
$$

Where $[H(\omega)]$ is the frequency response function, $\left[S_{F F}(\varpi)\right]$ is the cross power spectra of the input forces.

Similarly with the frequency response function of the traditional input-output system, in operational modal analysis, the cross power matrix of the output signals can 
also be modeled by the form of Right-Matrix Fraction Description model,

$$
\hat{S}_{i}(\omega)=F_{i}(\omega) G^{-1}(\omega)
$$

Where $F_{i}(\omega)$ is the numerator polynomial, and $G(\omega)$ is the common denominator polynomial, which are respectively defined as

$$
F_{i}(\omega)=\sum_{k=0}^{p} \Omega_{k}(\omega) \beta_{i k}, \quad G(\omega)=\sum_{k=0}^{p} \Omega_{k}(\omega) \alpha_{k}
$$

Where $p$ is the order of the polynomials, $\Omega_{k}(\omega)=e^{-j \omega T_{s} k}$ is the polynomial basis function, $\beta_{i k}$ and $\alpha_{k}$ sare the realvalued coefficients to be estimated. The real-valued coefficients $\beta_{i k}$ and $\alpha_{k}$ can be estimated by maximum likelihood estimator.

\section{Correlation for port crane structure model updating}

Correlation can be considered as the critical step to assess the quality of the analytical model of port crane structure. Before updating an analytical model, it is a common practice to compare the experimental and analytical data sets to obtain some insight as to whether both sets are in reasonable agreement so that updating is at all possible. According to the characteristics of port crane structures, in this paper, two correlation techniques are proposed to evaluate the agreement between the analytical modal data and experimental modal data.

\subsection{Frequency correlation}

The frequency accuracy of the experimental modal model could be controlled within $0.1 \%$, so frequency correlation is the most common and simplest method to compare the experimental modal model and the analytical ones. If the plot of the experimental values against analytical ones lies off a straight line of slope 1, the two modal models are perfectly correlated. A percentage difference of experimental and analytical models can be defined as shown in Eq. (4).

$$
C_{f i}=\frac{\omega_{e i}-\omega_{a i}}{\omega_{e i}} \times 100 \%
$$

Where $\omega_{e i}$ and $\omega_{a i}$ are respectively the experimental analytical frequencies of $i$ - th mode.

In engineering practice, it is usually required $\left|C_{f}\right| \leq$ $5 \%$. The accuracy of the analytical modal model could be judged only by the frequency correlation for the sparse frequency structure, but it's not enough for the large scale structure modal model, especially dense frequency ones. In that case, the modal assurance criterion (MAC) should be used also.

\subsection{Modal assurance criterion}

Modes pairing and modes matching are the most critical tasks when the model updating is based on modal testing data especially for port crane structures, so this paper utilized the modal assurance criterion (MAC) to pair and compare analytical and experimental mode shapes. MAC is easy to apply and does not require mass and stiffness matrices, and also it does not need to scale the experimental mode shapes, which is especially important for port crane structure model updating, because the operational experimental mode shapes cannot be absolutely scaled. MAC is defined by:

$$
M A C_{i}=\frac{\left(\varphi_{a i}{ }^{T} \varphi_{e i}\right)^{2}}{\left(\varphi_{a i}{ }^{T} \varphi_{a i}\right)^{2}\left(\varphi_{e i}{ }^{T} \varphi_{e i}\right)^{2}}
$$

where $\varphi_{e i}, \varphi_{a i}$ are respectively the paired experimental and analytical eigenmode.

The value of MAC is bound between 0 and 1 , a value of 1 means a perfect correlation, and a value of 0 means that the two modes do not show any correlation. In practice, the criterion is that high MAC values $(>0.7)$ are for a good correlation and value less than 0.05 for uncorrelated modes.

\section{Selection of the main factor based on sensitivity analysis}

The original port crane structure FE model is parametric modeled by the physical parameters such as material or geometrical or boundary properties etc. Usually there are a lot of physical parameters in a port crane structure FE model, during the model updating, the selected physical parameters to be modified should not only represent the uncertainty of the original model, but also be sensitive to the structure outputs, which are called the main factors. The main factors could be determined by the sensitivity analysis of the physical parameters [20-23].

Let the port crane structure be modelled by an undamped system, and the natural frequency sensitivity with respect to each parameter considered can be approxi- 
mated from the following equation,

$$
\frac{\partial \omega_{i}}{\partial a_{m}}=\frac{1}{2 \omega_{i}} \varphi_{i}^{T}\left(\frac{\partial K}{\partial a_{m}}-\omega_{i}^{2} \frac{\partial M}{\partial a_{m}}\right) \varphi_{i}
$$

where, $\omega_{i}$ and $\varphi_{i}$ are thei-th mode of the natural frequency and mode shape respectively, and $a_{m}$ is the m-th parameter which should be updated.

\section{Model updating of port crane structure}

\subsection{Optimization objective}

The problem of the parametric model updating is combined with the optimization problem together, the modal updating process is really the process of findinga set of main factors, that can be defined as optimal. Port crane structure model updating problem is the dynamic FE model multi-frequency optimization problem, which aims at minimizing the modal data which are calculated by analytical model and identified by experimental model according to the frequency correlation and MAC. The objective function can be defined as,

$$
O(a)=\sum_{i=1}^{k} w_{i \omega}\left(\frac{\omega_{e i}-\omega_{a i}(a)}{\omega_{e i}}\right)^{2}+\sum_{i=1}^{k} w_{i M A C}\left(1-M A C_{i}\right)
$$

where $w_{i \omega}$ and $w_{i M A C}$ are the weights of the $i$-th mode of frequency and MAC respectively. For port crane modal data identified by the operational analysis, the lower the mode is, the more confidence the result is, so in this paper, it is set that $w_{i \omega}=w_{i M A C}=1 / \omega_{e i}$.

\subsection{Optimization algorithm}

Eq. (7) is a constrained minimization problem, port crane structure multi-objective optimization model can be solved by zero-order optimization algorithm and firstorder optimization algorithm, All the two algorithms both need to use the penalty functions. The zero-order algorithm which achieves the global optimal solution requires only the values of the dependent variables and not their derivatives, which makes the zero-order algorithm cost less computation time but less accuracy. The first-order algorithm which achieves the local optimal solution calculates and makes use of derivative information, which is more computationally demanding and more accurate. In this paper, the zero-order and first-order combination algorithm is proposed to get the final accurate FE model of port crane structures.
For the zero-order algorithm, the dependent variables are first replaced with approximations by means of least squares fitting and the constrained minimization problem is converted to an unconstrained problem using penalty functions which are showed in Eq. (8). Minimization is then performed in every iteration on the approximated penalized function until convergence is achieved or termination is indicated.

$$
\min F(a, P)=O+O_{0} P\left[\sum_{m=1}^{p} A\left(a_{m}\right)+\sum_{i=1}^{n} D\left(d_{i}\right)\right]
$$

where $F(a, P)$ represents the unconstrained objective function that varies with the design parameters and the response surface parameter $P, A$ and $D$ are respectively the penalty functions used to enforce design parameter constrains and state variable constrains. The reference objective function value $O_{0}$ is introduced to achieve consistence unit. For the unconstrained problem Eq. (8) can be solved by the sequential unconstrained minimization technique.

For the first-order algorithm, the constrained problem statement can be transformed into an unconstrained one using penalty functions as,

$$
X(a, u)=\frac{O}{O_{0}}+\sum_{m=1}^{p} P_{a}\left(a_{m}\right)+u\left[\sum_{i=1}^{n} P_{d}\left(d_{i}\right)\right]
$$

where $X(a, u)$ is the dimensionless unconstrained objective function, $P_{a}, P_{d}$ are the penalties applied to the constrained design and state variables and $O_{0}$ refers to the reference objective function value, which is selected from the current group of design sets; $u$ is a response surface parameter.

In the first-order optimization, derivatives are formed for the objective function and state variable penalty functions, and various steepest descent and conjugate direction searches are performed during each iteration until convergence is reached. Each iteration is composed of sub iterations that include search direction and gradient computations.

The optimization stratagem in this paper is, finding a set of global optimal solutions less accurate by zero-order algorithm first, and then using the less accurate solutions as the initial value and carrying out the accurately calculation by the first-order algorithm to find the accurate global solution finally.

\subsection{Convergence}

Convergence is assumed when comparing the current iterations design set $(k+1)$ to the previous $(k)$ set and the optimal (o) set as show in Eq. (10), in which $\varepsilon$ is the objec- 
tive function tolerance. Various steepest descent and conjugated direction searches are performed during each iteration, until the convergence is reached.

$$
\left\{\begin{array}{l}
\left\|O\left(a^{(k+1)}\right)-O\left(a^{(k)}\right)\right\|<\varepsilon \\
\left\|\left(a^{(k+1)}\right)-O\left(a^{(o)}\right)\right\|<\varepsilon
\end{array}\right.
$$

\section{Case study}

A case study is carried out on a scaled port crane (see Figure 1). The basic functions of the scaled port crane (see Figure 1) include: hoisting, opening and closing of the grab, traveling and traversing of the crane etc. The metal structure of the prototype includes fore and back beam, seaside and land side frames, double forestay/backstay bars, $\mathrm{T}$ frame, landside platform etc.

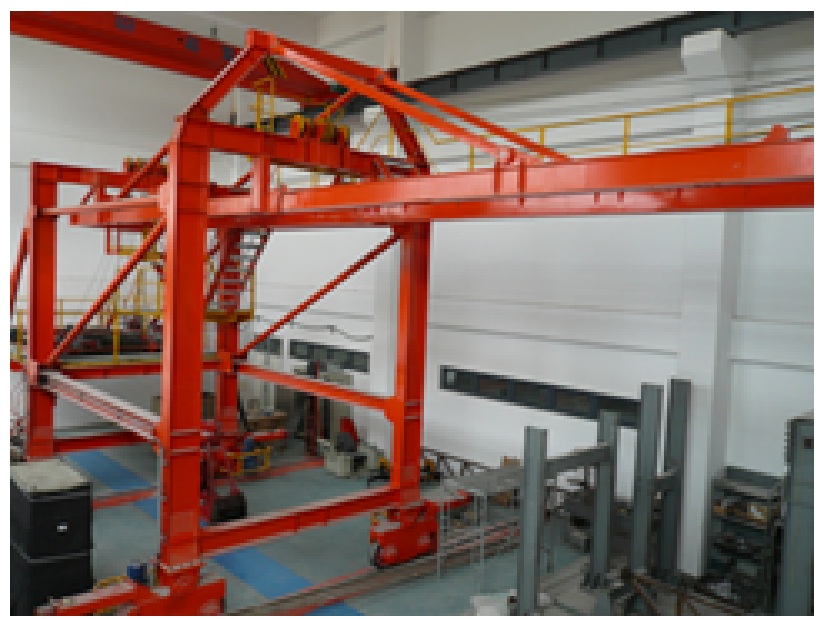

Figure 1: Photograph of the scaled port crane

The operational modal parameters of the scaled port crane are identified by the PolyMax method under the crane travelling excitation with 2 different trolley positions (positon1: parking, position2: forefront).

The equipments used for the operational vibration measurements include a 48-channel LMS data acquisition system with signal amplifier and conditioner, signal cables and B\&K accelerometers (4507\&4524). In order to acquire 3 directions vibration of the structure, 84 accelerometers (measurement points) were placed at 34 locations on the structure. 2 test setups were conceived to cover all the planned testing location. 3 measurement points were selected as references which were fixed during each test setup (Figure 4). The sampling frequency on site was $50 \mathrm{~Hz}$.
The parameters of first 6 modes of the scaled port crane are identified by the output-only data.

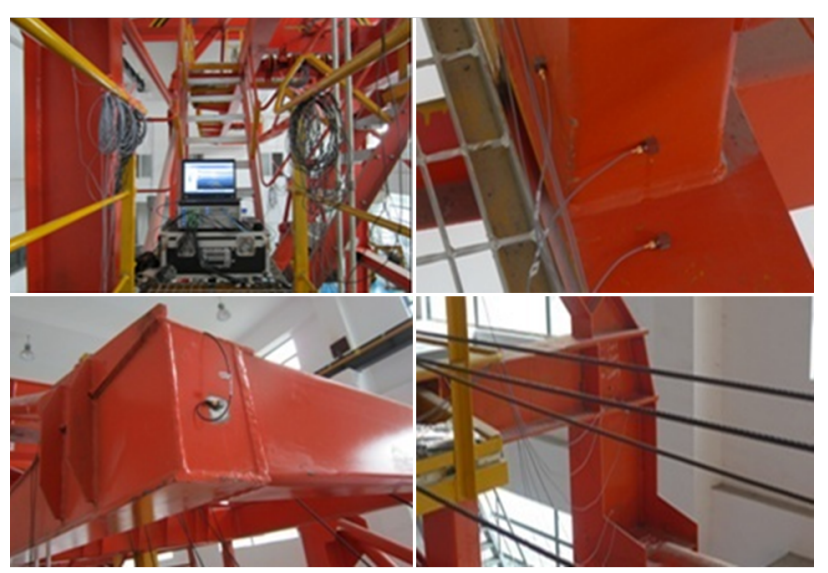

Figure 2: The testing site and equipments

Comparison of the natural frequencies between the experimental model and the original FE model of the crane can be found in Table 1 (trolley position 1). It shows that, the errors of mode 1 and mode 6 were higher than 5\%, which are usually unacceptable in engineering applications.

There are 20 pre-selected design parameters (11 geometry parameters, 3 material parameters and 6 boundary parameters etc.) in the original FE model because of model simplifying or knowledge lacking. Figure 4 shows the parameters sensitivity analysis by utilizing Eq. (6). It can be seen that 2 geometry parameters and 6 boundary parameters of the 20 pre-selected parameters have the relatively larger sensitivity values than others, which means they are much more sensitive to the objective. So in this study, these 8 parameters (g6, $g 7$ and b1to b6) which represent for the thickness parameters and the fore and back frame legs' constrain stiffness in $\mathrm{x}, \mathrm{y}, \mathrm{z}$ direction, respectively, are selected as the main factors for FE model updating.

The final modal parameters after FE model updating are showed in Figure 4, Figure 4(a) shows the differences between $\mathrm{FE}$ and experimental frequencies are all reduced to below $2 \%$, and it is clearly seen that all pair points are close to the diagonal, especially, the errors on the 1st, 4th and 6th mode frequency, they are all below $0.5 \%$. Figure 4(b) shows that the correlation of mode shape is also improved as all MAC values are over 0 . 88. It can be seen that the model updating result is a significant improvement comparing to the original FE model's. It has to be figured out, the accuracy of frequency of the updated model is much higher than the accuracy of mode shape, which can be attributed to two main reasons. One is due to the 
Table 1: Comparison of the natural frequencies between the experimental model and the original FE model (Position 1)

\begin{tabular}{llllll}
\hline Mode & $\omega_{e}(\mathrm{~Hz})$ & $\begin{array}{l}\omega_{a} \\
(\mathrm{~Hz})\end{array}$ & $\begin{array}{l}\text { Feq. } \\
\text { Errors } \\
(\%)\end{array}$ & $\begin{array}{l}\mathrm{MAC}_{i} \\
(\%)\end{array}$ & Mode Shape \\
\hline 1 & 1.034 & 1.096 & 6.03 & 94.18 & 1st cross flexural mode of the overall unit \\
2 & 1.688 & 1.657 & 1.82 & 95.12 & 1st cross flexural mode of the beam and frame \\
3 & 2.284 & 2.372 & 3.87 & 91.34 & 1st longitudinal mode of the overall unit \\
4 & 4.118 & 4.241 & 2.99 & 86.12 & 2nd cross flexural mode of the overall unit \\
5 & 5.129 & 5.323 & 3.79 & 80.87 & 3rd cross flexural mode of the overall unit \\
6 & 5.862 & 6.341 & 8.17 & 83.35 & 1st vertical mode of the overall unit \\
\hline
\end{tabular}

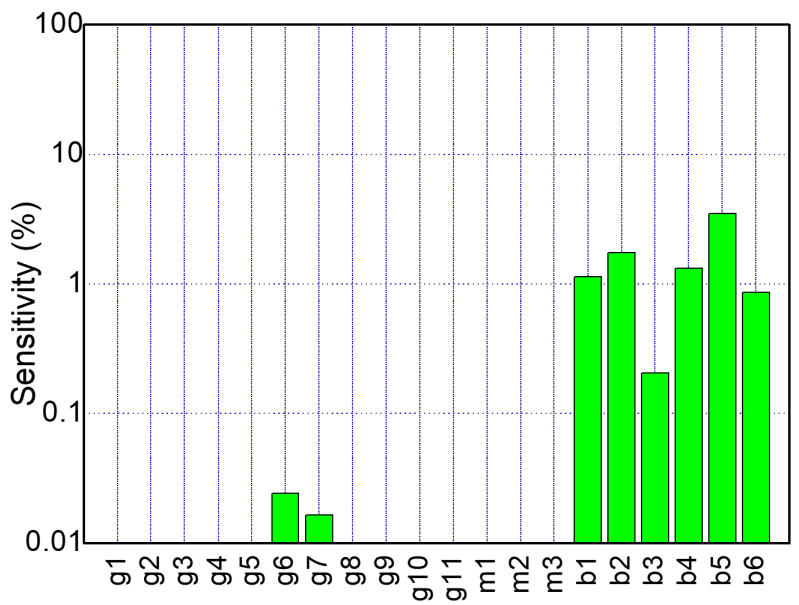

Figure 3: Parameters sensitivity analysis

structural modal parameters estimating from the experimental model which are based on the operational modal analysis instead of white noise, because the actual system input is always color noise, which caused the errors in modal parameters, especially in modal shapes. The other one is due to the inherent errors in simplification in the FE modeling, which could result in a greater impact on the high-ordered mode shape.

The structural modal parameters are dependent on systematic mass matrix and stiffness matrix. When the trolley is in different positions on port crane girder, the distribution of mass matrix and stiffness matrix will be different, which could cause the variation of the modal parameters of port crane. Figure 5 shows the difference in the frequency and MAC values among the first 6 modes identified by the operational modal analysis. The maximum difference in frequency is as high as $7 \%$ and the maximum difference in MAC values is $3 \%$, which indicates the variation of modal parameters caused by the trolley position is not negligible for port crane.

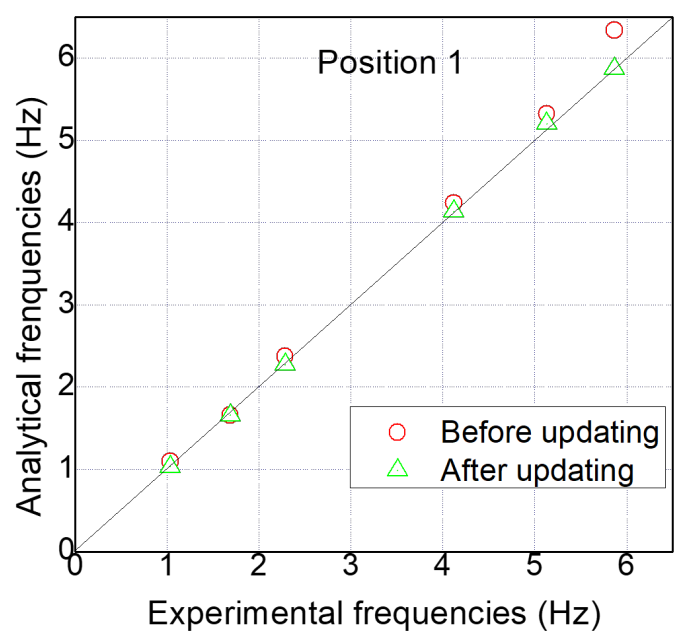

(a)

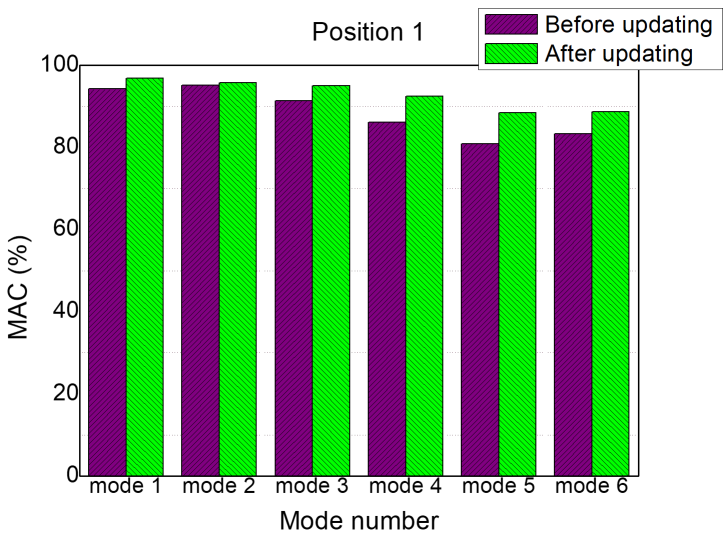

(b)

Figure 4: Modal parameters of the updated FE model (Position 1)

In order to verify the accuracy of the FE models of port crane with the updated design parameters for different trolley positions, the design parameters in the original $\mathrm{FE}$ 


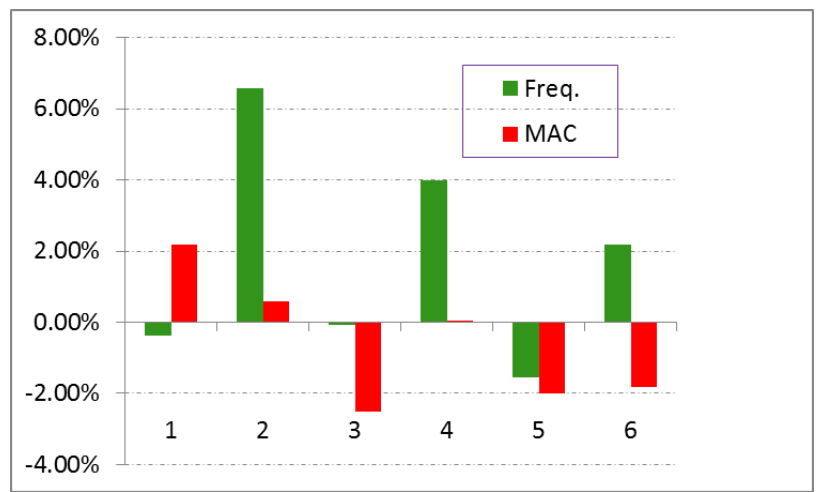

Figure 5: Differences of the frequency and MAC between position 1 and position 2
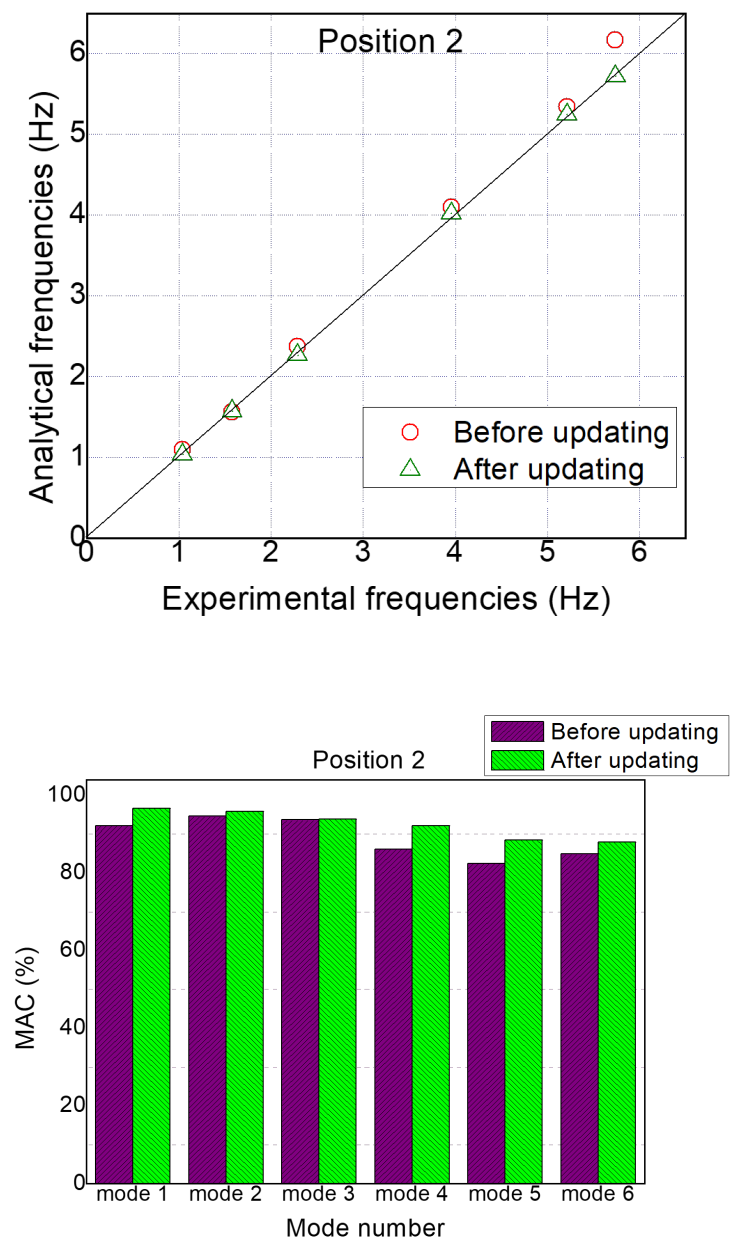

Figure 6: Modal parameters of the updated FE model (position 2)

model in position 2 is replaced by the design parameters in the updated model in position 1 to get the updated model for position 2 and also the structural modal parameters, which is shown in Figure 6. It is clear to see the modal pa- rameters in the updated model has been greatly improved compared to the original FE model, the improvement is as much as the updated FE model for position 1 (see Figure 7). So, for port crane structures, the updated design parameters from the FE model with single trolley position can be directly applied to the original FE model with different trolley positions, which will improve the updating efficiency of FE models of port crane under all working conditions.
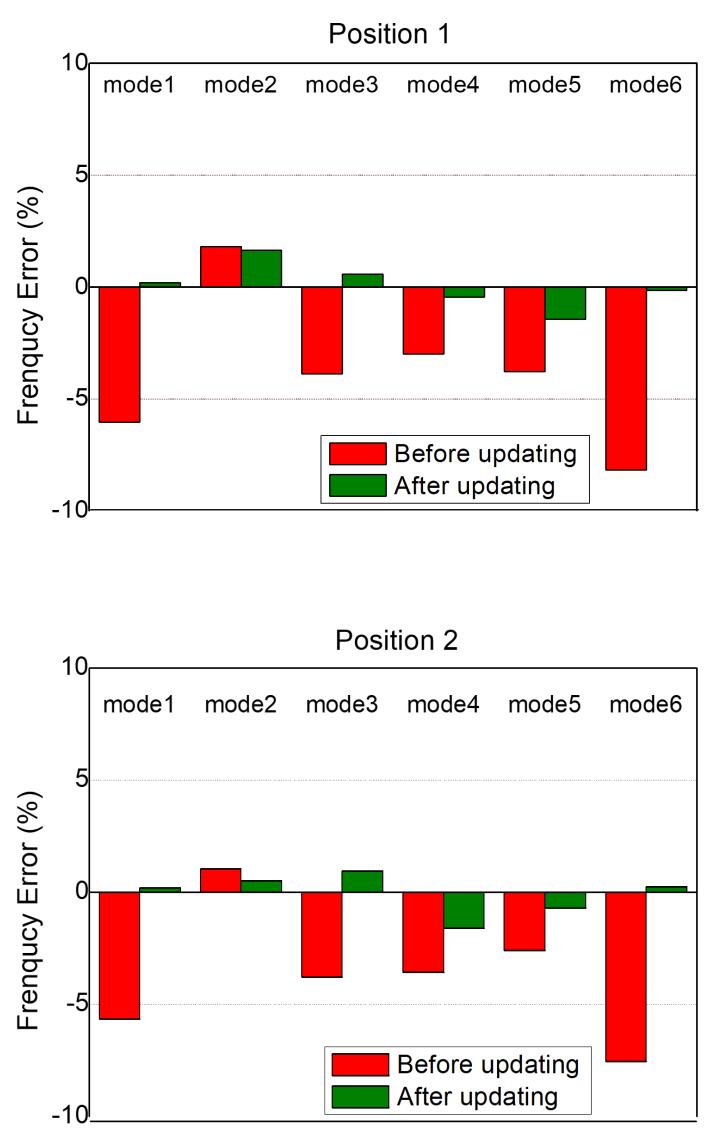

Figure 7: Frequency error of the updated FE model

\section{Conclusion}

Model updating of port crane structures needs approximation of the FE models according to the experiment models and results. In this study, port crane structure model updating is achieved by adopting the sensitivity based parametric FE model updating technique combined with the modal parameters identified by the operational modal analysis under ambient excitation. The objective function 
is established and the model is updated according to frequency correlation and modal assurance criterion (MAC) together, which can avoid the mismatching of mode shape caused by frequency approximation during model updating. The zero-order/first-order combination algorithm is adopted to optimize iteration, which can avert the optimization process stuck in local optimal solutions. The research results indicate that, the principles put forward in this paper can be applied to solve the model updating problems for large port crane structures, and the methodology adopted in the paper can help to achieve relatively satisfactory accuracy of the structural FE model, particularly for the structural frequency updating. The positions of the trolley can influence the modal parameters of port crane structure, the updated design parameters from the FE model with single trolley position can be used as the updated design parameters for port crane structures under all working conditions, which can achieve satisfactory updating results while saving lots of updating time.

Acknowledgement: This research was supported by the Science and Technology Commission of Shanghai Municipality (No. 17DZ1101401, No. 17DZ1101102, No. 18DZ1100901).

\section{References}

[1] Shu F., Mi W., Li X., Zhao N., Mi C., Yang X., A double-population genetic algorithm for asc loading sequence optimization in automated container terminals, J. Coastal Res., 2015, 73, 64-70.

[2] Bian Z., Yang Y., Mi W., Mi C. Dispatching electric agvs in automated container terminals with long travelling distance, J. Coastal Res., 2015, 73, 75-81.

[3] Zhao N., Shen Y., Xia M.,Jin J. A novel strategy for stowage planning of 40 feet containers in container terminals, J. Mar. Sci. Tech. Taiwan, 2016, 24(1), 61-74.

[4] Mi C., Shen Y., Mi W., Huang Y., Ship identification algorithm based on 3D point cloud for automated ship loaders, J. Coastal. Res., 2015, 73, 28-34.

[5] Mi C., Zhang Z., Huang Y., Shen Y., A fast automated vision system for container corner casting recognition, J. Mar. Sci. Tech. Taiwan, 2016, 24(1), 54-60.

[6] Nehete D.V., Modak S.V., Gupta K., Structural fe model updating of cavity systems incorporating vibro-acoustic coupling, Mech. Syst. Signal Pr., 2015, 50-51, 362-379.
[7] Simoen E., Roeck G.D., Lombaert G., Dealing with uncertainty in model updating for damage assessment: A review, Mech. Syst. Signal Pr., 2015, 56-57(56), 123-149.

[8] Rabi B.R.M., Nagaraj P., Finite element model updating of a space vehicle first stage motor based on experimental test results, Aerosp. Sci. Technol., 2015, 45, 422-430.

[9] Polanco N.R., May G., Hernandez E.M., Finite element model updating of semi-composite bridge decks using operational acceleration measurements, Eng. Struct., 2016, 126, 264-277.

[10] Jafarkhani R., Masri S.F., Finite element model updating using evolutionary strategy for damage detection, Comput.-Aided Civ. Inf., 2011, 26(3), 207-224.

[11] Zordan T., Briseghella B., Liu T., Finite element model updating of a tied-arch bridge using douglas-reid method and rosenbrock optimization algorithm, J. Traf. Transp. Eng. (Eng. Ed.), 2014, 1(4), 280-292.

[12] Berman A., Mass matrix correction using an incomplete set of measured modes, Aiaa J., 2012, 17(17), 1147-1148.

[13] Sanayei M., Arya B., Santini E.M., Sara W., Significance of modeling error in structural parameter estimation, Comput.-Aided Civ. Inf., 2010, 16(1), 12-27.

[14] Fox R.L., Kapoor M.P., Rates of change of eigenvalues and eigenvectors, Aiaa J., 1968, 6(12), 2426-2429.

[15] Mottershead J.E., Link M., Friswell M.I., The sensitivity method in finite element model updating: A tutorial, Mech. Syst. Signal Pr., 2011, 25(7), 2275-2296.

[16] James G.H.I., Carne T.G., Lauffer J.P., The natural excitation technique (next) for modal parameter extraction from operating wind turbines, Nasa Sti./Recon Technical Report N, 1993, 93(4), 260-277.

[17] Cauberghe B., Guillaume P., Verboven P., Parloo E., Vanlanduit S., A poly-reference implementation of the maximum likelihood complex frequency-domain estimator and some industrial applications, 2004.

[18] El-Kafafy M., Guillaume P., Peeters B., Modal parameter estimation by combining stochastic and deterministic frequencydomain approaches, Mech. Syst. Signal Pr., 2013, 35(1-2), 52 68.

[19] Friswell M.I., Mottershead J.E. Physical understanding of structures by model updating,2001.

[20] Klymchuk T., Regularizing algorithm for mixed matrix pencils, Appl. Math. Nonlin. Sci., 2017, 2(1), 123-130.

[21] Yang A., Han Y., Pan Y., Xing H., Li J., Optimum surface roughness prediction for titanium alloy by adopting response surface methodology, Res. Phys., 2017, 7, 1046-1050.

[22] Brown T., Du S., Eruslu H., Sayas F.J., Analysis of models for viscoelastic wave propagation, Appl. Math. Nonlin. Sci., 2018, 3, 55-96.

[23] Gao W., Wang Y., Basavanagoud B., Jamil M.K., Characteristics studies of molecular structures in drugs,Saudi Pharm. J., 2017, 25(4), 580-586. 